\title{
Factors associated with use of assistive walking devices among older adults in Brazil
}

\section{Fatores associados ao uso de tecnologia assistiva para auxiliar na mobilidade entre idosos no Brasil}

\author{
Flavia Cristina Drumond Andrade ${ }^{1}$ (D), Nessa Steinberg ${ }^{2}$ (D), Shondra L. Clay ${ }^{3}$ (D), Reginald Alston ${ }^{4}$ (D) \\ 'School of Social Work, University of Illinois at Urbana-Champaign - Champaign (IL), USA. \\ ${ }^{2}$ Department of Epidemiology, Johns Hopkins Bloomberg School of Public Health-Baltimore (MD), USA. \\ ${ }^{3}$ School of Interdisciplinary Health, Northern Illinois University - Dekalb (IL), USA. \\ ${ }^{4}$ Department of Kinesiology and Community Health, University of Illinois at Urbana-Champaign - Champaign (IL), USA.
}

How to cite: Andrade FCD, Steinberg N, Clay SL, Alston R. Factors associated with use of assistive walking devices among older adults in Brazil. Cad Saúde Colet, 2021;29(esp.):130-143. https://doi.org/10.1590/1414-462X202199010420

\begin{abstract}
Background: Walking devices and other forms of assistive technology (AT) can benefit older adults by supporting mobility and social interactions, but usage outside of high-income countries is generally low. Objective: To examine the factors associated with AT use and whether AT use is associated with higher levels of social participation among older adults in Brazil. Method: The 2013 Brazilian National Health Survey interviewed 23,815 individuals 60 years or older. Descriptive and logistic regression analyses were used to examine AT use, including canes and walkers, to assist with walking and social participation. Results: Among older adults with mobility difficulty, $34.0 \%(95 \% \mathrm{Cl} 31.2-36.9)$ reported using AT. Prevalence of the use of AT for walking increases with age: $21.4 \%$ of those $60-69$ years reported using AT while $58.5 \%$ of those 90 years or older did. AT was negatively associated with participation in social activities. Conclusion: Our analyses focused on older adults with mobility limitations who need appropriate transportation and environment adaptations to engage socially. Contrary to studies in more developed countries, among Brazilians, AT use is negatively associated with social interactions. The resulting confinement seems to lead to social isolation.
\end{abstract}

Keywords: assistive devices; walking; health of the elderly; social participation; Brazil.

\section{Resumo}

Introdução: Os dispositivos de tecnologia assistiva (TA) podem ajudar idosos na mobilidade e nas interações sociais, mas o uso fora de países de alta renda é geralmente baixo. Objetivo: Analisar os fatores associados ao uso de TA e se o uso de TA está associado a níveis mais elevados de participação social entre idosos no Brasil. Método: A Pesquisa Nacional de Saúde - PNS 2013 no Brasil entrevistou 23.815 indivíduos com 60 anos ou mais. Análises descritivas e de regressão logística foram utilizadas para examinar a utilização de TA para auxiliar na mobilidade e participação social. Resultados: Entre idosos com dificuldade de locomoção, 34,0\% (95\% IC 31,2-36,9) relataram uso de TA. A prevalência do uso de TA para se locomover aumenta com a idade: $21,4 \%$ dos 60-69 anos relataram usar TA enquanto a proporção aumenta para $58,5 \%$ entre os de 90 anos ou mais. Uso de TA está negativamente associado à participação em atividades sociais. Conclusão: Entre idosos no Brasil com limitações de mobilidade que necessitam de adaptações adequadas de transporte e meio ambiente para engajar socialmente, 0 uso de TA foi associado negativamente às interações sociais. Esse resultado difere de estudos em países mais desenvolvidos.

Palavras-chave: dispositivos assistivos; caminhada; saúde do idoso; participação social; Brasil.

Study carried out at University of Illinois at Urbana-Champaign - Champaign (IL), USA.

Correspondence: Flavia Cristina Drumond Andrade. E-mail: fandrade@illinois.edu

Financial support: none.

Conflict of interests: nothing to declare.

Received on: Sept. 20, 2019. Accepted on: Dec. 26, 2019
This is an Open Access article distributed under the terms of the Creative Commons Attribution License, which permits unrestricted use, distribution, and reproduction in any medium, provided the original work is properly cited. 


\section{INTRODUCTION}

Assistive technology (AT) such as canes, wheelchairs, walkers, and crutches can be essential for enhancing the lives of persons with mobility issues ${ }^{1}$, supporting independence ${ }^{2,3}$ and mobility ${ }^{4}$. Studies conducted in developed countries show that AT use can increase participation in social activities ${ }^{1,5}$. Such participation correlates with high quality of life $\mathrm{e}^{6,7}$. Nevertheless, individuals with mobility issues may face difficulties adapting to elements of AT, from the characteristics of the device (e.g., weight or size) to physical environment barriers (e.g., access to public transportation, difficulty maneuvering in private and public spaces) to reluctance to use them due to negative social perceptions ${ }^{1}$. There are also financial barriers to purchasing these devices, and in many environments patients and their families bear heavy costs to acquire them ${ }^{8}$. Finally, the use of devices may encourage older adults to enter spaces they would not otherwise, leaving them vulnerable to crime?

Most research on the impact of AT has focused on high-income countries; Marasinghe et al..$^{10}$ identified only four studies exploring AT use in Brazil. This is a major gap because Brazil has been experiencing fast-paced growth of its older adult population - in 2015, $12 \%$ of the population was 60 years or older and this proportion is expected to reach $19 \%$ in $2030^{11}$. Issues related to mobility have become increasingly urgent in the country because old age is a major risk factor for mobility problems. Figures from 2013 indicate that $1.3 \%$ of Brazilians (approximately 2.6 million people) have a physical disability, including $1.6 \%$ of those in the Northeast and 3.3\% of adults 60 years of age or older ${ }^{12}$. Since 1988, Brazil has assured access to AT for Brazilians who need them ${ }^{13}$, but barriers may remain and the conditions of the country may affect the impact of their use on patient outcomes.

The current study examines AT use by individuals with mobility limitations using a national representative data of adults 60 years and older from the 2013 Brazilian National Health Survey (Pesquisa Nacional de Saúde, PNS). We expand on the work developed by Malta et al. ${ }^{12}$ by focusing on older adults who have mobility limitations and their use of AT. To increase knowledge about the value and potentialities of AT, this study follows the conceptual framework used by Peterson et al. ${ }^{14}$, which examines differentials in AT use based on the disablement model and Theory of Planned Behavior. We use an adapted version that includes the following domains: sociodemographic characteristics, health conditions, and social environmental factors. The guiding research questions of this study are ${ }^{1}$ : Among older adults with mobility difficulties, what are the domains associated with AT use? And 2) Among older adults with higher levels of mobility limitations, is AT use associated with higher levels of social participation?

\section{METHOD}

\section{Survey and setting}

Individual-level data came from the 2013 PNS. This household-based survey collects information such as educational attainment, health status, health behaviors, and access and use of health services. It employs a multistage, probability sampling design. First, researchers obtained a subsample of the Brazilian Census Bureau (Instituto Brasileiro de Geografia e Estatistica, IBGE) master sample of the 2010 Integrated System of Household Surveys (Sistema Integrado de Pesquisas Domiciliares). Primary sampling units (PSU) were based on census tracts, then the PSUs were categorized by size and the number of tracts randomly selected from each tract determined by category. Researchers then randomly selected households from the selected tracts. In the final stage, one adult (18 years or older) was randomly chosen among all adults in these households ${ }^{15}$.

The PNS questionnaire is divided into three parts. The first two parts consist of information on the household characteristics and the health status of all household members. The third part concerns the selected adult and it includes questions about chronic conditions, lifestyle, and oral health ${ }^{15}$. Detailed information about the PNS, including questionnaires, survey design, and 
datasets, can be found at the the Fundação Instituto Oswaldo Cruz (Fiocruz) website (www.pns. fiocruz.br). The Brazilian Ministry of Health (Ministério da Saúde, MS) has carried out the PNS in partnership with the Brazilian Census Bureau. The National Committee for Ethics in Research (Comissão Nacional de Ética em Pesquisas) approved the PNS in June 2013. All individuals included in this study consented to participate.

\section{Participants}

The 2013 PNS interviewed 23,815 individuals 60 years or older, but only a subset were included in the sample of selected adults $(n=11,177)$, who responded to all questions used in this study. Among those, 8,283 reported having no difficulty moving around and 2,894 reported at least some difficulty (1,342 mild, 837 medium, 474 intense, and 241 are immobile). Among those who reported having no difficulty, 76 used AT to assist them with mobility and ambulation. As discussed by Cornman et al. ${ }^{16}$, some individuals use AT to move around, but report no difficulty. These individuals may be using AT as a preventative measure to avoid injuries, particularly from falls ${ }^{16}$. Excluding these individuals, the analysis would fail to include a segment of the population who are adapting successfully to $\mathrm{AT}^{16}$. Therefore, this study considers the 2,970 older adults who reported any level of difficulty with mobility and/ or who reported using AT, such as canes, walkers, and wheelchairs. Among those, $136 \mathrm{had}$ missing data on hypertension status, diabetes, and/or race. The final sample for AT use (aim 1) is limited to 2,834 with complete data on selected variables. There were no differences in age, AT use, or mobility difficulties between those dropped due to missing data and the sample with complete data, although a higher proportion of men had missing data than women $(p<0.001)$. A subsample of 675 individuals with complete data and more severe mobility limitations were used to examine social participation, as explained below.

\section{AT use}

Use of AT was based on whether participants used any device such as a cane, wheelchair, walker, or any other equipment to assist with mobility (based on respondents' answers to the question, "Do you use a device such as cane, crutches, wheelchair, walker or other equipment to aid in locomotion?" " $\mathrm{O}(\mathrm{A}) \mathrm{sr}(\mathrm{a})$ usa algum recurso como bengala, muleta, cadeira de rodas, andador ou outro equipamento para auxiliar a locomoção?"). Those who responded affirmatively were considered to be using AT and those who responded negatively were considered not to be using it.

\section{Social participation}

Participants were asked if they participated in social activities, such as clubs, community or religious groups, and centers for older adults. Those who responded affirmatively were considered as being socially engaged and those negatively as not.

\section{Sociodemographic characteristics}

The sociodemographic domain included a dichotomous variable for female (male as the reference group), a continuous variable for age in years; race (White, Black, Pardo [which is Brazil's term for mixed-race people], and those of Asian descent and Indigenous); education (no education, primary, secondary, and some college or more), region of residence (South, Southeast, Midwest, Northeast, North); and a dichotomous variable for private health insurance. Those who reported having more than one health insurance plan were classified as having private health insurance.

\section{Health conditions}

Number of health conditions is based on self-reported data on previous medical diagnosis "Has a doctor given you a diagnosis of [health condition]?" "Algum médicojá lhe deu o diagnóstico 
de [doença]?") The data include responses to questions in which the health condition was cancer, diabetes, hypertension, heart disease, stroke, arthritis, or renal disease. Those who responded affirmatively were considered as having the chronic condition and those who responded negatively as not having it. Self-reported health was based on the participants' ratings. Those who reported fair or poor health were categorized as having poor health. Participants were also asked if they had fallen, fractured a hip, and/or if they were hospitalized in the last 12 months. Information on body mass index (BMI) was also included. Body weight and height were measured by trained examiners. BMI was calculated as $\mathrm{kg} / \mathrm{m}^{2}$. Three $\mathrm{BMI}$ categories were defined according to the adult criteria: underweight (BMI<18.5), normal (BMI 18.5-24.9); overweight (BMI 25.0-29.9); and obese $(\mathrm{BMI} \geq 30)$. Finally, we included variables related to visual and hearing impairments. Psychosocial variables included self-reported information on depression diagnosis.

\section{Social environment}

The social environment included measures related to marital status (married, separated or divorced, widowed, or single), average family and friendship networks, whether or not the participant receives informal care to assist with mobility issues, and exposure to violence.

\section{Statistical analysis}

Descriptive statistics are presented in Table 1. Bivariate analyses were used to examine whether users and non-users differ across sociodemographic, health, and social environment domains. Chi-squared tests were used for the categorical variables and the $t$-test for the continuous variables. Table 2 presents the results of the multivariate logistic regressions that examine the likelihood of AT use among participants with mobility limitations (aim 1). We provide results for three hierarchical models. Model 1 includes the sociodemographic domain, model 2 adds health domain variables, and model 3 adds social environmental variables. Next, we examine whether AT use is associated with higher social participation among individuals who reported having severe mobility limitations (aim 2). Sample weights and study design were considered using a complex sampling design in all data analyses. All statistical analyses were done using STATA SE 14.2.

\section{RESULTS}

Among older adults who reported having mobility difficulties, $34.0 \%(95 \% \mathrm{Cl} 31.2-36.9)$ reported using AT, including canes, walkers, wheelchairs and other devices. The mean age of the sample was 74 years $(95 \% \mathrm{Cl} 73.2-74.3)$ and women predominated (62.5\%). Those with mobility difficulties had low levels of education, with $41 \%$ reporting no education and the other $45.6 \%$ reporting only primary education. About a third had private health insurance in addition to access to the public health system. The most common chronic conditions were hypertension (61.0\%), arthritis (28.0\%), diabetes (25.6\%), heart disease (17.3\%), stroke (12.5\%), and cancer (7.1\%). Hospitalization in the last 12 months was common (17.1\%), and many older adults in the sample reported having fallen recently (13.1\%). In terms of impairments, visual limitations (20.1\%) were more common than hearing limitations (8.0\%). Regarding the social environment, most were unmarried (54.2\%), with average family network of 3 and friendship network of 2 (Table 1 ).

As in prior studies, we found that prevalence of AT use for walking increases with age. Among those $60-69$ years, $21.4 \%$ reported using AT, and this proportion increased to $58.5 \%$ among those 90 years or older, but there were no differences by sex, race, region, or private insurance. However, there was higher use of AT among those with lower education in bivariate analyses. There was higher use of AT among those with history of heart disease, stroke, and cancer and among those who self-reported being in poor health. Recent hospitalizations and falls, being underweight, and having a previous diagnosis of depression likewise correlated with AT use. A higher proportion of those using AT also reported receiving informal care related to assistance with walking (Table 1). 
Table 1. Characteristics of Sample Population, Stratified by AT Use, Brazilian National Health Survey, 2013

\begin{tabular}{|c|c|c|c|c|}
\hline & $\begin{array}{c}\text { No use } \\
(n=1,881)\end{array}$ & Use $(n=953)$ & $\begin{array}{c}\text { Total } \\
(\mathrm{n}=\mathbf{2 , 8 3 4 )}\end{array}$ & $p$-value \\
\hline \multicolumn{5}{|l|}{ Sociodemographic } \\
\hline Age groups & & & & $<0.0001$ \\
\hline $60-69$ & 43.2 & 22.8 & 36.3 & \\
\hline 70-79 & 35.9 & 35.1 & 35.6 & \\
\hline $80-89$ & 17.1 & 31.8 & 22.1 & \\
\hline$\geq 90$ & 3.8 & 10.3 & 6.0 & \\
\hline Sex & & & & 0.7752 \\
\hline Male & 36.5 & 39.4 & 37.5 & \\
\hline Female & 63.5 & 60.6 & 62.5 & \\
\hline Race & & & & 0.5888 \\
\hline White & 53.1 & 56.0 & 54.1 & \\
\hline Black & 9.2 & 9.5 & 9.3 & \\
\hline Yellow & 1.0 & 0.6 & 0.9 & \\
\hline Pardo & 36.6 & 33.8 & 35.7 & \\
\hline Education & & & & 0.0169 \\
\hline No education & 38.7 & 46.7 & 41.4 & \\
\hline Primary or secondary incomplete & 47.4 & 42.1 & 45.6 & \\
\hline Secondary complete & 7.6 & 5.0 & 6.7 & \\
\hline Some college or more & 6.2 & 6.2 & 6.2 & \\
\hline Region of residence & & & & 0.5126 \\
\hline North & 5.6 & 3.9 & 5.0 & \\
\hline Northeast & 27.0 & 28.2 & 27.4 & \\
\hline Midwest & 5.1 & 6.3 & 5.5 & \\
\hline Southeast & 45.3 & 44.2 & 45.0 & \\
\hline South & 17.0 & 17.4 & 17.2 & \\
\hline Private insurance & & & & 0.7752 \\
\hline No & 70.0 & 70.9 & 70.3 & \\
\hline Yes & 30.0 & 29.1 & 29.7 & \\
\hline \multicolumn{5}{|l|}{ Health conditions } \\
\hline Heart disease & & & & 0.0181 \\
\hline No & 84.6 & 79.1 & 82.7 & \\
\hline Yes & 15.5 & 20.9 & 17.3 & \\
\hline High blood pressure & & & & 0.2439 \\
\hline No & 37.8 & 41.2 & 39.0 & \\
\hline
\end{tabular}


Table 1. Continued...

\begin{tabular}{|c|c|c|c|c|}
\hline & $\begin{array}{c}\text { No use } \\
(n=1,881)\end{array}$ & Use ( $n=953)$ & $\begin{array}{c}\text { Total } \\
(n=2,834)\end{array}$ & $p$-value \\
\hline Yes & 62.2 & 58.8 & 61.0 & \\
\hline Arthritis & & & & 0.0916 \\
\hline No & 73.7 & 68.9 & 72.0 & \\
\hline Yes & 26.4 & 31.1 & 28.0 & \\
\hline Diabetes & & & & 0.12 \\
\hline No & 75.9 & 71.6 & 74.4 & \\
\hline Yes & 24.2 & 28.4 & 25.6 & \\
\hline Lung disease & & & & 0.1519 \\
\hline No & 94.4 & 91.4 & 93.3 & \\
\hline Yes & 5.6 & 8.6 & 6.7 & \\
\hline Stroke & & & & $<0.0001$ \\
\hline No & 92.6 & 77.7 & 87.5 & \\
\hline Yes & 7.4 & 22.3 & 12.5 & \\
\hline Cancer & & & & 0.0007 \\
\hline No & 94.8 & 89.2 & 92.9 & \\
\hline Yes & 5.2 & 10.8 & 7.1 & \\
\hline Self-rated health (fair/poor) & & & & 0.0039 \\
\hline No & 76.7 & 68.9 & 74.1 & \\
\hline Yes & 23.3 & 31.1 & 26.0 & \\
\hline Hospitalization & & & & $<0.0001$ \\
\hline No & 87.0 & 75.0 & 82.9 & \\
\hline Yes & 13.0 & 25.0 & 17.1 & \\
\hline Falls in the last 12 months & & & & $<0.0001$ \\
\hline No & 90.7 & 79.5 & 86.9 & \\
\hline Yes & 9.3 & 20.5 & 13.1 & \\
\hline Fracture & & & & 0.0965 \\
\hline No & 98.4 & 96.2 & 97.6 & \\
\hline Yes & 1.6 & 3.8 & 2.4 & \\
\hline Body Mass Index & & & & 0.0123 \\
\hline Underweight & 1.9 & 5.0 & 3.0 & \\
\hline Normal & 32.0 & 32.4 & 32.1 & \\
\hline Overweight & 36.0 & 37.0 & 36.3 & \\
\hline Obese & 30.1 & 25.6 & 28.6 & \\
\hline Hearing impairment & & & & 0.0764 \\
\hline
\end{tabular}


Table 1. Continued...

\begin{tabular}{|c|c|c|c|c|}
\hline & $\begin{array}{c}\text { No use } \\
(n=1,881)\end{array}$ & Use ( $n=953)$ & $\begin{array}{c}\text { Total } \\
(n=2,834)\end{array}$ & $p$-value \\
\hline No & 93.0 & 90.1 & 92.0 & \\
\hline Yes & 7.0 & 10.0 & 8.0 & \\
\hline Visual impairment & & & & 0.2128 \\
\hline No & 81.0 & 77.7 & 79.9 & \\
\hline Yes & 19.0 & 22.3 & 20.1 & \\
\hline Depression & & & & 0.009 \\
\hline No & 83.3 & 89.7 & 85.5 & \\
\hline Yes & 16.7 & 10.3 & 14.5 & \\
\hline \multicolumn{5}{|l|}{ Social environment } \\
\hline Marital status & & & & 0.0014 \\
\hline Married & 49.8 & 38.1 & 45.8 & \\
\hline Separated or divorced & 6.4 & 7.1 & 6.6 & \\
\hline Widowed & 32.1 & 41.2 & 35.2 & \\
\hline Single & 11.7 & 13.7 & 12.4 & \\
\hline Family network (mean) & 3.5 & 3.3 & 3.4 & 0.548 \\
\hline Friendship network (mean) & 2.2 & 2.4 & 2.2 & 0.553 \\
\hline Receives walking help & & & & $<0.0001$ \\
\hline No & 95.8 & 72.0 & 87.7 & \\
\hline Yes & 4.2 & 28.0 & 12.3 & \\
\hline Exposure to violence & & & & 0.4737 \\
\hline No & 98.5 & 99.0 & 98.7 & \\
\hline Yes & 1.5 & 1.0 & 1.4 & \\
\hline
\end{tabular}

\section{AT use}

Table 2 presents the results of the logistic regression models with AT use as the outcome variable. In Model 1, controlling for sociodemographic characteristics, older age was associated with higher use of AT. In Model 2, health status variables were added to the model. Older age remained an important determinant of AT use, but those living in the North region, which is poorer, more isolated and less densely populated, were less likely to report AT use than those in the Southeast region. Use of AT in other regions did not differ from the Southeast region. Those who reported having a previous diagnosis of arthritis, diabetes, stroke, or cancer, or who reported being in poor health, recently hospitalized, or having recently fallen had higher odds of using AT; however, hypertension was negatively associated with AT use. Individuals with a previous diagnosis of depression were less likely to use AT. When social environment predictors were added to the model, the results indicated that being single, divorced, or separated are associated with higher use of AT, as well as having a larger friendship network. Individuals who also received informal care for walking were more likely to use AT. In addition, older age was associated with higher AT use. Those living in the North region were less likely to use 
Table 2. Odds-ratios and 95\% confidence intervals for AT use in Brazil, 2013

\begin{tabular}{|c|c|c|c|c|c|c|c|c|c|}
\hline \multirow{2}{*}{ VARIABLES } & \multicolumn{3}{|c|}{ Model 1} & \multicolumn{3}{|c|}{ Model 2} & \multicolumn{3}{|c|}{ Model 3} \\
\hline & OR & $95 \% \mathrm{Cl}$ & $p$ & OR & $95 \% \mathrm{Cl}$ & $p$ & OR & $95 \% \mathrm{Cl}$ & $p$ \\
\hline \multicolumn{10}{|c|}{ Sociodemographic } \\
\hline \multicolumn{10}{|l|}{$\begin{array}{l}\text { Age groups } \\
\text { (ref. 60-69 years) }\end{array}$} \\
\hline 70-79 & 1.86 & {$[1.38,2.50]$} & $<0.001$ & 1.81 & {$[1.34,2.44]$} & 0.0001 & 1.72 & {$[1.25,2.36]$} & 0.0008 \\
\hline $80-89$ & 3.49 & {$[2.51,4.86]$} & $<0.001$ & 3.29 & {$[2.33,4.65]$} & $<0.001$ & 2.82 & {$[1.92,4.15]$} & $<0.001$ \\
\hline$\geq 90$ & 5.07 & {$[3.14,8.19]$} & $<0.001$ & 5.49 & {$[3.43,8.78]$} & $<0.001$ & 3.77 & {$[2.23,6.37]$} & $<0.001$ \\
\hline \multicolumn{10}{|l|}{ Sex (ref. Male) } \\
\hline Female & 0.86 & {$[0.65,1.13]$} & 0.2744 & 1.07 & {$[0.80,1.42]$} & 0.6557 & 0.93 & {$[0.69,1.27]$} & 0.6553 \\
\hline \multicolumn{10}{|l|}{ Race (ref. White) } \\
\hline Black & 1.11 & {$[0.70,1.76]$} & 0.6565 & 0.99 & {$[0.61,1.62]$} & 0.9712 & 1.11 & {$[0.66,1.85]$} & 0.7038 \\
\hline Yellow & 0.68 & {$[0.26,1.78]$} & 0.4346 & 0.73 & {$[0.28,1.89]$} & 0.5139 & 0.79 & {$[0.28,2.26]$} & 0.6599 \\
\hline Pardo & 0.99 & {$[0.73,1.34]$} & 0.9476 & 1.09 & {$[0.80,1.48]$} & 0.5995 & 1.12 & {$[0.82,1.54]$} & 0.4820 \\
\hline \multicolumn{10}{|l|}{$\begin{array}{l}\text { Education (ref. no } \\
\text { education) }\end{array}$} \\
\hline $\begin{array}{l}\text { Primary or } \\
\text { secondary } \\
\text { incomplete }\end{array}$ & 0.85 & {$[0.63,1.13]$} & 0.2638 & 0.81 & {$[0.60,1.10]$} & 0.1707 & 0.84 & {$[0.62,1.13]$} & 0.2432 \\
\hline $\begin{array}{l}\text { Secondary } \\
\text { complete }\end{array}$ & 0.68 & {$[0.42,1.10]$} & 0.1146 & 0.63 & {$[0.37,1.10]$} & 0.1029 & 0.64 & {$[0.38,1.10]$} & 0.1086 \\
\hline $\begin{array}{l}\text { Some college or } \\
\text { more }\end{array}$ & 0.95 & {$[0.55,1.61]$} & 0.8374 & 1.11 & {$[0.62,1.96]$} & 0.7332 & 1.12 & {$[0.64,1.98]$} & 0.6931 \\
\hline \multicolumn{10}{|l|}{$\begin{array}{l}\text { Region of } \\
\text { residence } \\
\text { (ref. Southeast) }\end{array}$} \\
\hline North & 0.72 & {$[0.46,1.15]$} & 0.1672 & 0.53 & {$[0.32,0.87]$} & 0.0132 & 0.50 & {$[0.29,0.85]$} & 0.0104 \\
\hline Northeast & 0.97 & {$[0.70,1.33]$} & 0.8295 & 0.93 & {$[0.66,1.29]$} & 0.6479 & 0.87 & {$[0.63,1.19]$} & 0.3787 \\
\hline Midwest & 1.34 & {$[0.89,2.01]$} & 0.1673 & 1.09 & {$[0.69,1.73]$} & 0.7035 & 1.05 & {$[0.67,1.66]$} & 0.8178 \\
\hline South & 1.17 & {$[0.80,1.72]$} & 0.4183 & 1.03 & {$[0.69,1.54]$} & 0.8962 & 1.10 & {$[0.72,1.68]$} & 0.6650 \\
\hline \multicolumn{10}{|l|}{$\begin{array}{l}\text { Private insurance } \\
\text { (ref. no) }\end{array}$} \\
\hline Yes & 0.95 & {$[0.68,1.33]$} & 0.7564 & 0.95 & {$[0.67,1.33]$} & 0.7454 & 1.07 & {$[0.76,1.49]$} & 0.7101 \\
\hline \multicolumn{10}{|l|}{ Health conditions } \\
\hline Heart disease & & & & 1.28 & {$[0.92,1.80]$} & 0.1461 & 1.33 & {$[0.94,1.87]$} & 0.102 \\
\hline $\begin{array}{l}\text { High blood } \\
\text { pressure }\end{array}$ & & & & 0.72 & {$[0.56,0.94]$} & 0.0141 & 0.73 & {$[0.56,0.95]$} & 0.0179 \\
\hline Arthritis & & & & 1.32 & {$[1.01,1.72]$} & 0.0444 & 1.32 & {$[1.00,1.74]$} & 0.0527 \\
\hline Diabetes & & & & 1.45 & {$[1.08,1.95]$} & 0.0142 & 1.45 & {$[1.09,1.93]$} & 0.0116 \\
\hline Lung disease & & & & 1.19 & {$[0.67,2.10]$} & 0.5565 & 0.89 & {$[0.49,1.60]$} & 0.6921 \\
\hline Stroke & & & & 4.00 & {$[2.83,5.67]$} & $<0.001$ & 2.93 & {$[2.09,4.11]$} & $<0.001$ \\
\hline Cancer & & & & 1.95 & {$[1.22,3.12]$} & 0.0052 & 2.05 & {$[1.20,3.52]$} & 0.0091 \\
\hline $\begin{array}{l}\text { Self-rated health } \\
\text { (fair/poor) }\end{array}$ & & & & 1.41 & {$[1.05,1.88]$} & 0.0206 & 1.32 & {$[0.97,1.78]$} & 0.0728 \\
\hline
\end{tabular}


Table 2. Continued...

\begin{tabular}{|c|c|c|c|c|c|c|c|c|c|}
\hline \multirow{2}{*}{ VARIABLES } & \multicolumn{3}{|c|}{ Model 1} & \multicolumn{3}{|c|}{ Model 2} & \multicolumn{3}{|c|}{ Model 3} \\
\hline & OR & $95 \% \mathrm{Cl}$ & $p$ & OR & $95 \% \mathrm{Cl}$ & $p$ & OR & $95 \% \mathrm{Cl}$ & $p$ \\
\hline Hospitalization & & & & 1.84 & {$[1.33,2.55]$} & 0.0002 & 1.49 & {$[1.07,2.08]$} & 0.0199 \\
\hline $\begin{array}{l}\text { Falls in the last } 12 \\
\text { months }\end{array}$ & & & & 1.97 & {$[1.33,2.92]$} & 0.0007 & 1.89 & {$[1.29,2.78]$} & 0.0011 \\
\hline Fracture & & & & 1.01 & {$[0.28,3.57]$} & 0.9933 & 0.79 & {$[0.17,3.67]$} & 0.7652 \\
\hline \multicolumn{10}{|l|}{$\begin{array}{l}\text { Body Mass Index } \\
\text { (ref. normal) }\end{array}$} \\
\hline Underweight & & & & 2.42 & {$[1.27,4.60]$} & 0.0071 & 2.9 & {$[1.55,5.41]$} & 0.0008 \\
\hline Overweight & & & & 1.15 & {$[0.86,1.55]$} & 0.3423 & 1.21 & {$[0.89,1.63]$} & 0.2261 \\
\hline Obese & & & & 1.12 & {$[0.78,1.60]$} & 0.5369 & 1.15 & {$[0.80,1.66]$} & 0.4347 \\
\hline $\begin{array}{l}\text { Hearing } \\
\text { impairment }\end{array}$ & & & & 1.1 & {$[0.70,1.72]$} & 0.6836 & 1.15 & {$[0.73,1.81]$} & 0.5381 \\
\hline Visual impairment & & & & 1.05 & {$[0.74,1.48]$} & 0.7899 & 1.15 & {$[0.82,1.63]$} & 0.4141 \\
\hline Depression & & & & 0.61 & {$[0.40,0.92]$} & 0.0178 & 0.55 & {$[0.37,0.84]$} & 0.0048 \\
\hline \multicolumn{10}{|l|}{$\begin{array}{l}\text { Marital status (ref. } \\
\text { married) }\end{array}$} \\
\hline $\begin{array}{l}\text { Separated or } \\
\text { divorced }\end{array}$ & & & & & & & 1.94 & {$[1.11,3.39]$} & 0.0195 \\
\hline Widowed & & & & & & & 1.32 & {$[0.95,1.84]$} & 0.0995 \\
\hline Single & & & & & & & 1.64 & {$[1.08,2.49]$} & 0.0190 \\
\hline Family network & & & & & & & 0.99 & {$[0.96,1.02]$} & 0.3694 \\
\hline Friendship network & & & & & & & 1.04 & {$[1.01,1.07]$} & 0.0065 \\
\hline $\begin{array}{l}\text { Receives walking } \\
\text { help }\end{array}$ & & & & & & & 6.02 & {$[3.86,9.38]$} & $<0.001$ \\
\hline $\begin{array}{l}\text { Exposure to } \\
\text { violence }\end{array}$ & & & & & & & 1.38 & {$[0.49,3.91]$} & 0.5414 \\
\hline Constant & 0.33 & {$[0.21,0.53]$} & $<0.001$ & 0.19 & {$[0.09,0.28]$} & $<0.001$ & 0.12 & {$[0.07,0.21]$} & $<0.001$ \\
\hline Observations & 2,834 & & & 2,834 & & & 2,834 & & \\
\hline
\end{tabular}

AT. The chronic conditions associated with higher AT use were arthritis, diabetes, stroke, and cancer; however, hypertension was negatively associated with AT use. Older adults with recent hospitalizations or falls, or who were underweight were more likely to use AT.

\section{Social participation}

Table 3 presents the results of aim 2, which focuses on whether the use of AT helps older adults with more severe mobility difficulties to engage in social activities. In model 1 , individuals who used AT were less likely to be engaged in social activities than those who did not use AT. Within this sample, those 70-90 years old were more likely to participate in social events than those aged 60-69 years. Primary or secondary education attainment also correlated with higher participation. Pardo individuals, those with private health insurance, and those who live in the South region were more likely to be engaged in social activities. Model 2 includes the health characteristics. Use of AT remained negatively associated with engagement in social activities. However, having a respiratory condition or being in poor health was associated with lower 
Table 3. Odds-ratios and 95\% confidence intervals for social participation and AT use in Brazil, 2013

\begin{tabular}{|c|c|c|c|c|c|c|c|c|c|}
\hline \multirow{2}{*}{ VARIABLES } & \multicolumn{3}{|c|}{ Model 1} & \multicolumn{3}{|c|}{ Model 2} & \multicolumn{3}{|c|}{ Model 3} \\
\hline & OR & $95 \% \mathrm{Cl}$ & $p$ & OR & $95 \% \mathrm{Cl}$ & $p$ & OR & $95 \% \mathrm{Cl}$ & $p$ \\
\hline Assistive device use & 0.48 & {$[0.27,0.86]$} & 0.0134 & 0.48 & {$[0.25,0.93]$} & 0.0285 & 0.65 & {$[0.33,1.28]$} & 0.2153 \\
\hline \multicolumn{10}{|l|}{ Sociodemographics } \\
\hline \multicolumn{10}{|l|}{$\begin{array}{l}\text { Age groups } \\
\text { (ref. 60-69 years) }\end{array}$} \\
\hline $70-79$ & 2.22 & {$[1.06,4.69]$} & 0.0356 & 1.8 & {$[0.79,4.14]$} & 0.1631 & 1.62 & {$[0.69,3.80]$} & 0.2625 \\
\hline $80-89$ & 2.56 & {$[1.15,5.71]$} & 0.022 & 2.42 & {$[1.10,5.35]$} & 0.0286 & 2.47 & {$[1.10,5.55]$} & 0.0293 \\
\hline$\geq 90$ & 0.82 & {$[0.25,2.63]$} & 0.7327 & 0.55 & {$[0.13,2.29]$} & 0.4107 & 0.62 & {$[0.15,2.61]$} & 0.5173 \\
\hline \multicolumn{10}{|l|}{ Sex (ref. Male) } \\
\hline Female & 1.34 & {$[0.66,2.72]$} & 0.4206 & 1.35 & {$[0.64,2.86]$} & 0.4324 & 1.5 & {$[0.63,3.58]$} & 0.3619 \\
\hline \multicolumn{10}{|l|}{ Race (ref. White) } \\
\hline Black & 1.02 & {$[0.45,2.33]$} & 0.9546 & 0.86 & {$[0.35,2.11]$} & 0.7465 & 1.06 & {$[0.40,2.78]$} & 0.9099 \\
\hline Yellow & 0.12 & {$[0.01,1.14]$} & 0.0644 & 0.12 & {$[0.01,1.24]$} & 0.0748 & 0.14 & {$[0.01,1.47]$} & 0.1017 \\
\hline Pardo & 2.77 & {$[1.15,6.67]$} & 0.0237 & 2.93 & {$[1.21,7.06]$} & 0.017 & 3.41 & {$[1.30,8.96]$} & 0.013 \\
\hline \multicolumn{10}{|l|}{$\begin{array}{l}\text { Education (ref. no } \\
\text { education) }\end{array}$} \\
\hline $\begin{array}{l}\text { Primary or } \\
\text { secondary } \\
\text { incomplete }\end{array}$ & 3.63 & {$[1.72,7.66]$} & 0.0008 & 2.84 & {$[1.49,5.39]$} & 0.0015 & 2.61 & {$[1.42,4.78]$} & 0.002 \\
\hline $\begin{array}{l}\text { Secondary } \\
\text { complete }\end{array}$ & 3.5 & {$[1.19,10.34]$} & 0.0235 & 2.78 & {$[0.99,7.80]$} & 0.0514 & 2.55 & {$[0.95,6.83]$} & 0.0619 \\
\hline $\begin{array}{l}\text { Some college or } \\
\text { more }\end{array}$ & 0.65 & {$[0.09,4.56]$} & 0.6656 & 0.48 & {$[0.06,4.15]$} & 0.5064 & 0.33 & {$[0.03,3.41]$} & 0.3528 \\
\hline \multicolumn{10}{|l|}{$\begin{array}{l}\text { Region of residence } \\
\text { (ref. Southeast) }\end{array}$} \\
\hline North & 2.49 & {$[0.80,7.74]$} & 0.1129 & 2.76 & {$[0.87,8.80]$} & 0.0858 & 2.65 & {$[0.77,9.09]$} & 0.1216 \\
\hline Northeast & 1.18 & {$[0.43,3.23]$} & 0.7521 & 1.31 & {$[0.60,2.85]$} & 0.4958 & 1.35 & {$[0.60,3.07]$} & 0.4704 \\
\hline Midwest & 1.73 & {$[0.42,7.20]$} & 0.449 & 1.9 & {$[0.52,6.94]$} & 0.328 & 1.55 & {$[0.39,6.17]$} & 0.5304 \\
\hline South & 5.01 & {$[2.08,12.07]$} & 0.0004 & 6.09 & {$[2.63,14.11]$} & 0 & 5.5 & {$[2.41,12.55]$} & 0.0001 \\
\hline \multicolumn{10}{|l|}{$\begin{array}{l}\text { Private insurance } \\
\text { (ref. no) }\end{array}$} \\
\hline Yes & 2.27 & {$[1.07,4.81]$} & 0.0319 & 2.18 & {$[1.09,4.36]$} & 0.0276 & 2.09 & {$[0.95,4.57]$} & 0.0655 \\
\hline \multicolumn{10}{|l|}{ Health conditions } \\
\hline Heart disease & & & & 1.63 & {$[0.72,3.69]$} & 0.24 & 1.6 & {$[0.71,3.60]$} & 0.2545 \\
\hline High blood pressure & & & & 1.44 & {$[0.72,2.89]$} & 0.306 & 1.71 & {$[0.90,3.26]$} & 0.1014 \\
\hline Arthritis & & & & 1.46 & {$[0.78,2.73]$} & 0.2416 & 1.36 & {$[0.75,2.46]$} & 0.3168 \\
\hline Diabetes & & & & 0.98 & {$[0.49,1.98]$} & 0.9602 & 0.96 & {$[0.46,1.99]$} & 0.9054 \\
\hline Lung disease & & & & 0.06 & {$[0.01,0.31]$} & 0.0007 & 0.07 & {$[0.01,0.29]$} & 0.0004 \\
\hline Stroke & & & & 1.61 & {$[0.74,3.51]$} & 0.2285 & 2.44 & {$[1.05,5.66]$} & 0.0384 \\
\hline Cancer & & & & 0.88 & {$[0.23,3.29]$} & 0.8461 & 0.82 & {$[0.22,3.09]$} & 0.7661 \\
\hline $\begin{array}{l}\text { Self-rated health } \\
\text { (fair/poor) }\end{array}$ & & & & 0.35 & {$[0.16,0.75]$} & 0.007 & 0.29 & {$[0.12,0.67]$} & 0.0039 \\
\hline
\end{tabular}


Table 3. Continued...

\begin{tabular}{|c|c|c|c|c|c|c|c|c|c|}
\hline \multirow{2}{*}{ VARIABLES } & \multicolumn{3}{|c|}{ Model 1} & \multicolumn{3}{|c|}{ Model 2} & \multicolumn{3}{|c|}{ Model 3} \\
\hline & OR & $95 \% \mathrm{Cl}$ & $p$ & OR & $95 \% \mathrm{Cl}$ & $p$ & OR & $95 \% \mathrm{Cl}$ & $p$ \\
\hline Hospitalization & & & & 0.57 & {$[0.26,1.28]$} & 0.1761 & 0.58 & {$[0.26,1.28]$} & 0.1763 \\
\hline $\begin{array}{l}\text { Falls in the last } 12 \\
\text { months }\end{array}$ & & & & 0.55 & {$[0.20,1.53]$} & 0.2502 & 0.47 & {$[0.15,1.45]$} & 0.1878 \\
\hline Fracture & & & & 2.12 & {$[0.14,32.79]$} & 0.5902 & 4.1 & {$[0.23,73.15]$} & 0.3359 \\
\hline \multicolumn{10}{|l|}{$\begin{array}{l}\text { Body Mass Index } \\
\text { (ref. normal) }\end{array}$} \\
\hline Underweight & & & & 1.96 & {$[0.72,5.35]$} & 0.189 & 1.94 & {$[0.68,5.54]$} & 0.2175 \\
\hline Overweight & & & & 0.85 & {$[0.40,1.80]$} & 0.6711 & 0.89 & {$[0.41,1.95]$} & 0.7746 \\
\hline Obese & & & & 1.09 & {$[0.46,2.59]$} & 0.8498 & 1.14 & {$[0.46,2.80]$} & 0.7777 \\
\hline Hearing impairment & & & & 0.97 & {$[0.35,2.70]$} & 0.948 & 0.8 & {$[0.29,2.24]$} & 0.6697 \\
\hline Visual impairment & & & & 1.79 & {$[0.81,3.96]$} & 0.1495 & 1.78 & {$[0.84,3.76]$} & 0.1331 \\
\hline Depression & & & & 0.59 & {$[0.28,1.24]$} & 0.161 & 0.58 & {$[0.27,1.23]$} & 0.153 \\
\hline \multicolumn{10}{|l|}{$\begin{array}{l}\text { Marital status } \\
\text { (ref. married) }\end{array}$} \\
\hline $\begin{array}{l}\text { Separated or } \\
\text { divorced }\end{array}$ & & & & & & & 0.27 & {$[0.09,0.77]$} & 0.0148 \\
\hline Widowed & & & & & & & 0.88 & {$[0.34,2.29]$} & 0.7896 \\
\hline Single & & & & & & & 0.53 & {$[0.19,1.50]$} & 0.2308 \\
\hline Family network & & & & & & & 0.9 & {$[0.84,0.97]$} & 0.0077 \\
\hline Friendship network & & & & & & & 1.06 & {$[0.99,1.15]$} & 0.1079 \\
\hline $\begin{array}{l}\text { Receives walking } \\
\text { help }\end{array}$ & & & & & & & 0.29 & {$[0.11,0.77]$} & 0.0131 \\
\hline $\begin{array}{l}\text { Exposure to } \\
\text { violence }\end{array}$ & & & & & & & 1.17 & {$[0.21,6.56]$} & 0.8605 \\
\hline Constant & 0.02 & {$[0.00,0.05]$} & 0 & 0.02 & {$[0.01,0.06]$} & 0 & 0.03 & {$[0.01,0.09]$} & 0 \\
\hline Observations & 675 & & & 675 & & & 675 & & \\
\hline
\end{tabular}

odds of social engagement. Model 3 includes the social environment variables. Results showed that AT use did not influence social engagement after controlling for social environment. Older adults with a higher family network or who received informal help for walking were less likely to be socially engaged. Those who were separated or divorced were less likely than married people to be engaged in social activities; however, those who were single did not differ from those who were married.

\section{DISCUSSION}

Our results indicate that the use of AT differs across sociodemographic groups, health status, and social environments. In general, among individuals with mobility limitation, use of AT is higher at older ages and among those with more disabling chronic conditions, such as stroke, cancer, and diabetes. Older adults who are underweight, had fallen in the last 12 months, or who had been hospitalized are also more likely to use AT. This aligns with the fact that as individuals get older their health worsens, and they are more likely to use AT. We also examine 
whether AT use is associated with higher social participation. Among older adults who report having more severe mobility difficulties, the use of AT did not improve social participation. To the best of our knowledge, this is the first study to examine sociodemographic, health and social environmental factors associated with AT use and social participation of AT users in Brazil using a national representative sample.

Previous studies have found sex differences in AT use ${ }^{14,17}$, with women often more likely to present disability than men, but less likely to use devices. However, we found no sex differences in AT use in Brazil. We also accounted for socioeconomic differences. We found no educational differences in the use of AT, but residents in the North region are less likely to use AT, which may reflect regional differences in the availability of access to health care ${ }^{18}$.

In our study, older adults who have suffered falls or had a stroke were more likely to use AT. A previous study conducted in Brazil, based on a small sample of institutionalized older adults, found that wheelchair users reported fear of falls, lack of assistance for walking, and fatigue ${ }^{19}$. However, reduced physical abilities, such as having visual or hearing impairments, which are often associated with older adults feeling unsteady when walking, were not associated with AT use. These findings highlight how the use of AT, such as wheelchairs and canes, have the potential to assist older adults who face important changes in their health status, such as falls or stroke, to adapt to their environments ${ }^{16,20}$. Nonetheless, this adaptation does not occur without difficulties resulting from physical, environmental and social barriers. In addition, effective AT use requires instruction and monitoring from medical professionals ${ }^{20}$.

Participation in social activities is often associated with higher quality of life ${ }^{6}$. We examined whether the use of AT could improve participation in social activities, but our results indicate no statistically significant differences between users and non-users. This contrasts with studies that have found that AT use improves social engagement ${ }^{1,5}$, but it is similar to another study that found that assistive devices in Brazil did not improve independence ${ }^{21}$. As highlighted in a systematic review of studies addressing the use of walking devices and social participation, AT use only seems to facilitate social participation and engagement when users integrate them into their everyday lives'. This finding suggests that older adults in Brazil, even if they use AT, may face additional barriers to engage in social activities or have difficulties integrating them into their everyday lives. Older adults, particularly those in poor health, seem to have lower social engagement in this sample. In addition, our analyses focused on those with more severe limitations and whose participation in social activities require not only adequate accommodations, such as AT use, but also appropriate transportation, public safety, environment adaptations, among others. In fact, as individuals become older and frailer, the use of public transportation becomes increasingly challenging in places like Brazil, because the system is generally of poor quality and not adapted to provide comfort and security to these individuals ${ }^{22}$. The country's infrastructure is limited and individuals using AT face several challenges, such as poorly maintained sidewalks. The study does not take Brazil's physical environment in terms of safety and public transportation into account in these measures, yet they greatly impact older adults' willingness and ability to engage socially. The data also do not provide information on longevity, which would show whether social engagement changes when AT use begins. Adults who are not socially engaged may be more likely to develop severe mobility issues.

On the other hand, results indicate that older adults with larger family networks and those who receive informal care for mobility issues are less likely to engage in social activities. It is possible that these older adults are more severely disabled and face more difficulty getting around. Another possibility is that, given their larger network, they feel less need to engage in further social activities.

The proportion of older adults who reported using devices to assist with their mobility problems is relatively low in Brazil, with almost two-thirds not using any device. In Brazil, health care is a universal human right, and access has become more equitable in recent decades ${ }^{23}$. In the last decade, Brazil introduced the "Viver sem limites" ("Life without limits") program, whose main objective is to guarantee disability rights, such as education, social inclusion, and accessibility. As part of this program, individuals with disability had access to special credit lines 
to purchase motorized wheelchairs. Another indication of the governmental commitment to improve the lives of individuals with disability was the "Lei Brasileira de Inclusão" ("Inclusion Law") of $2015^{24}$, which states the fundamental rights of education, transportation, and health. As a result of these policies and legislation, the number of individuals receiving AT equipment, such as wheelchairs, has increased ${ }^{25}$. However, there are several barriers that may still limit the use of AT in the country. First, empirical research has not clarified whether the recent programs have resolved issues with availability of $A T^{13,25}$ and adequate training of health professionals such that they properly prescribe AT to patients and effectively teach patients to use them ${ }^{26}$, which were issues identified prior to the introduction of these new programs. Second, the process by which practitioners assess patients and match AT to their needs does not include a full understanding of the context in which patients live ${ }^{24}$. Third, most homes require adaptations, such as widening of doors, for AT use ${ }^{27}$, but the public health care system does not cover the costs of these adaptations.

While this study is broadly generalizable to Brazil, given its use of national representative data, it has a few limitations. First, data are cross-sectional, which precludes causal inferences. Longitudinal studies are needed to examine how health and social changes influence AT use, adaptation, and social engagement. Second, health conditions were reported by either respondents or proxy respondents, which may be subject to biase ${ }^{28}$. In addition, data on health conditions do not include severity or duration, which could assist in better understanding AT use and social engagement. Data on cognitive health status are lacking, which may influence the results. Third, there are no data related to whether AT use was discontinued, which could be important for public policies. Fourth, there is no information as to whether people who obtained AT equipment use it, or to how well adapted to it they are.

Brazil has been facing rapid population aging, and the proportion of older adults vulnerable to social isolation is likely to increase in coming decades. We found differences in the use of AT in Brazil and, so far, AT does not seem to be associated with social participation among those with more severe limitations. If AT use is to assist older adults in their social lives, it should be appropriate for their needs and contexts. Individuals and families need to accept AT as a way to improve their lives, but they must be well adapted to social and physical environments.

\section{REFERENCES}

1. Bertrand K, Raymond M-H, Miller WC, Ginis KAM, Demers L. Walking aids for enabling activity and participation: a systematic review. Am J Phys Med Rehabil. 2017;96(12):894-903. http://dx.doi.org/10.1097/ PHM.0000000000000836. PMid:29176406.

2. Mann WC, Ottenbacher KJ, Fraas L, Tomita M, Granger CV. Effectiveness of assistive technology and environmental interventions in maintaining independence and reducing home care costs for the frail elderly: a randomized controlled trial. Arch Fam Med. 1999;8(3):210-7. http://dx.doi.org/10.1001/ archfami.8.3.210. PMid:10333815.

3. Edyburn DL. Efficacy of assistive technology interventions. Bingley: Emerald Group; 2015. Expanding the use of assistive technology while mindful of the need to understand efficacy; p. 1-12. http://dx.doi. org/10.1108/S2056-769320150000001001.

4. Clay SL, Alston R. The benefits of assistive technology use by persons with physical conditions: An examination of difficulty levels in areas of functioning. Technol Disabil. 2016;28(3):111-21. http://dx.doi. org/10.3233/TAD-160449.

5. Krishna S, Colbry D, Black J, Balasubramanian V, Panchanathan S, editors. A systematic requirements analysis and development of an assistive device to enhance the social interaction of people who are blind or visually impaired. In: Workshop on Computer Vision Applications for the Visually Impaired; 2008; San Diego, CA. New York: IEEE; 2008.

6. Ilunga Tshiswaka D, Loggins Clay S, Chiu C-Y, Alston R, Lewis A. Assistive technology use by disability type and race: exploration of a population-based health survey. Disabil Rehabil Assist Technol. 2016;11(2):12432. http://dx.doi.org/10.3109/17483107.2015.1090487. PMid:26414744.

7. World Health Organization. International classification of functioning, disability and health: ICF. Geneva: WHO; 2001. 
8. Garçon L, Khasnabis C, Walker L, Nakatani Y, Lapitan J, Borg J, et al. Medical and assistive health technology: meeting the needs of aging populations. Gerontologist. 2016;56(Suppl. 2):S293-302. http://dx.doi. org/10.1093/geront/gnw005. PMid:26994268.

9. Scherer HL, Reyns BW.Visible disabilities and risk of interpersonal victimization. In: Berry B, editor. Appearance bias and crime. New York: Bonnie Berry; 2019. 243 p. http://dx.doi.org/10.1017/9781108377683.014.

10. Marasinghe KM, Lapitan JM, Ross A. Assistive technologies for ageing populations in six low-income and middle-income countries: a systematic review. BMJ Innov. 2015;1(4):182-95. http://dx.doi.org/10.1136/ bmjinnov-2015-000065. PMid:26688747.

11. Economic Commission for Latin America and the Caribbean. Demographic observatory. Santiago: ECLAC; 2016.

12. Malta DC, Stopa SR, Canuto R, Gomes NL, Mendes VLF, Goulart BN, et al. Self-reported prevalence of disability in Brazil, according to the National Health Survey, 2013. Cien Saude Colet. 2016;21(10):3253-64. http://dx.doi.org/10.1590/1413-812320152110.17512016. PMid:27783798.

13. Mann WC, Mello MAF. Assistive technology use by the elderly in Brazil and the United States. Top Geriatr Rehabil. 2010;26(1):62-9. http://dx.doi.org/10.1097/TGR.0b013e3181cd695e.

14. Peterson LJ, Meng H, Dobbs D, Hyer K. Gender differences in mobility device use among U.S. older adults. J Gerontol B Psychol Sci Soc Sci. 2017;72(5):827-35. PMid:27495837.

15. Szwarcwald CL, Malta DC, Pereira CA, Vieira MLFP, Conde WL, Souza PR Jr, et al. National Health Survey in Brazil: design and methodology of application. Cien Saude Colet. 2014;19(2):333-42. http://dx.doi. org/10.1590/1413-81232014192.14072012. PMid:24863810.

16. Cornman JC, Freedman VA, Agree EM. Measurement of assistive device use: implications for estimates of device use and disability in late life. Gerontologist. 2005;45(3):347-58. http://dx.doi.org/10.1093/ geront/45.3.347. PMid:15933275.

17. Agree EM, Freedman VA, Sengupta M. Factors influencing the use of mobility technology in communitybased long-term care. J Aging Health. 2004;16(2):267-307. http://dx.doi.org/10.1177/0898264303262623. PMid:15030666.

18. Travassos C, Viacava F, Fernandes C, Almeida CM. Desigualdades geográficas e sociais na utilização de serviços de saúde no Brasil. Cien Saude Colet. 2000;5(1):133-49. http://dx.doi.org/10.1590/S141381232000000100012

19. Alves EF, Bezerra PP. Fatores associados ao uso de cadeira de rodas por idosos institucionalizados. Cien Saude Colet. 2017;22(11):3607-14. http://dx.doi.org/10.1590/1413-812320172211.14332016. PMid:29211166.

20. Hamzat T, Kobiri A. Effects of walking with a cane on balance and social participation among communitydwelling post-stroke individuals. Eur J Phys Rehabil Med. 2008;44(2):121-6. PMid:18418331.

21. Cruz DM, Emmel ML. Associations among occupational roles, independence, assistive technology, and purchasing power of individuals with physical disabilities. Rev Lat Am Enfermagem. 2013;21(2):484-91. http://dx.doi.org/10.1590/S0104-11692013000200003. PMid:23797540.

22. Martins M, Fatigati F, Vespoli T, Martins L, Pereira L, Martins M, et al. Influence of socioeconomic conditions on air pollution adverse health effects in elderly people: an analysis of six regions in Sao Paulo, Brazil. $J$ Epidemiol Community Health. 2004;58(1):41-6. http://dx.doi.org/10.1136/jech.58.1.41. PMid:14684725.

23. Macinko J, Lima-Costa MF. Horizontal equity in health care utilization in Brazil, 1998-2008. Int J Equity Health. 2012;11(1):33. http://dx.doi.org/10.1186/1475-9276-11-33. PMid:22720869.

24. Alves AC, Matsukura TS, Scherer MJ. Cross-cultural adaptation of the assistive technology device: predisposition assessment (ATD PA) for use in Brazil (ATD PA Br). Disabil Rehabil Assist Technol. 2017;12(2):160-4. http://dx.doi.org/10.1080/17483107.2016.1233294. PMid:27882819.

25. Maximo T, Clift L. Assessing service delivery systems for assistive technology in Brazil using HEART study quality indicators. Technol Disabil. 2016;27(4):161-70. http://dx.doi.org/10.3233/TAD-160438.

26. Alves ACJ, Emmel MLG, Matsukura TS. Formação e prática do terapeuta ocupacional que utiliza tecnologia assistiva como recurso terapêutico. Rev Ter Ocup Univ Sao Paulo. 2012;23(1):24-33. http://dx.doi. org/10.11606/issn.2238-6149.v23i1p24-33.

27. Mello MAF, editor. The need of assistive devices by older dependent people living in community. In Proceedings of International Conference of Aging, Disability and Independence (ICADI); 2006; St. Petersburg, Florida. Amsterdam: IOS Press; 2006.

28. Burgard SA, Chen PV. Challenges of health measurement in studies of health disparities. Soc Sci Med. 2014;106:143-50. http://dx.doi.org/10.1016/j.socscimed.2014.01.045. PMid:24561776. 